\title{
A prognostic staging system for light-chain amyloidosis using hepatic and renal indicator data from 1,064 Chinese patients
}

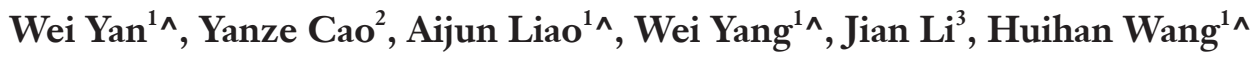 \\ ${ }^{1}$ Department of Hematology, Shengjing Hospital of China Medical University, Shenyang, China; ${ }^{2}$ Neusoft Research Institute, Northeastern \\ University, Shenyang, China; ${ }^{3}$ Department of Hematology, Peking Union Medical College Hospital, Chinese Academy of Medical Sciences \& \\ Peking Union Medical College, Beijing, China \\ Contributions: (I) Conception and design: W Yan, H Wang; (II) Administrative support: W Yan; (III) Provision of study materials or patients: A Liao, \\ W Yang, J Li, H Wang; (IV) Collection and assembly of data: W Yan; (V) Data analysis and interpretation: Y Cao; (VI) Manuscript writing: All \\ authors; (VII) Final approval of manuscript: All authors. \\ Correspondence to: Huihan Wang. Department of Hematology, Shengjing Hospital of China Medical University, 39 Huaxiang Road, Shenyang \\ 110000, China. Email: 240333079@qq.com.
}

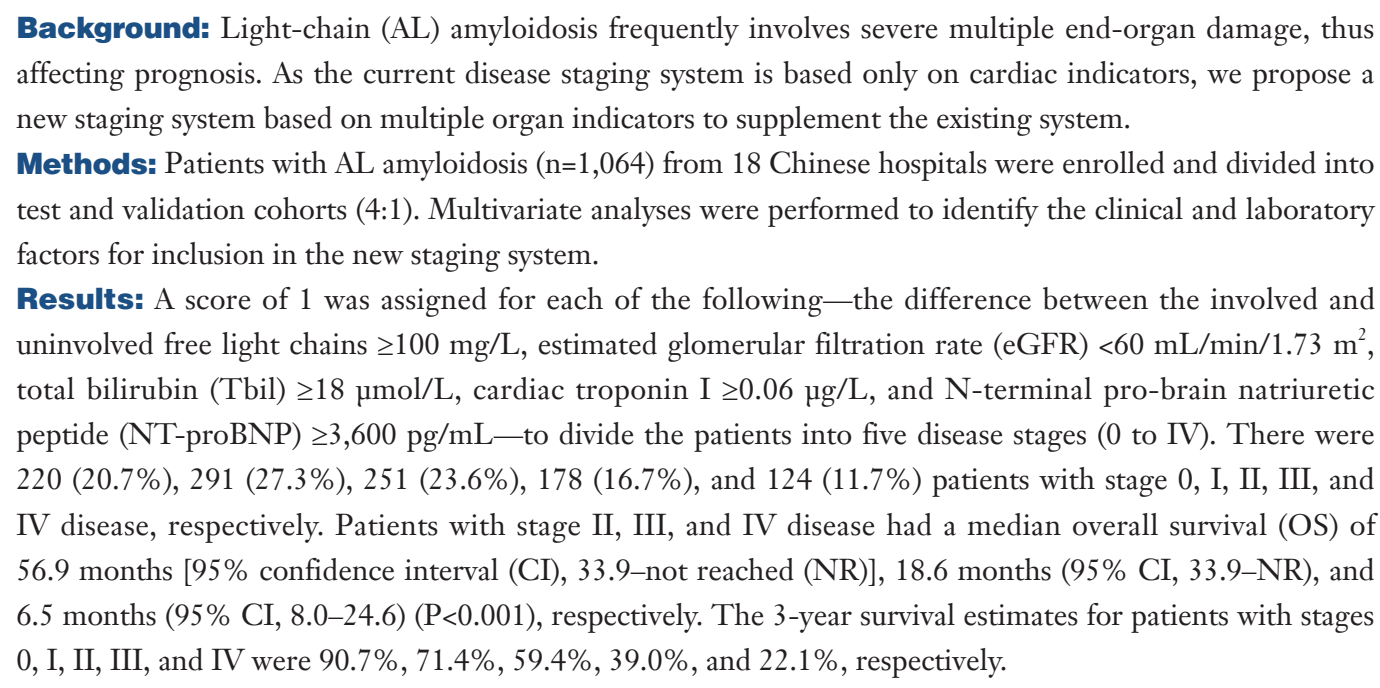

Conclusions: The new staging system has been developed that incorporates plasma cell-related characteristics in addition to cardiac, renal, and hepatic function parameters. It enhances the risk stratification of patients with AL amyloidosis and is useful when multiple organs are involved.

Keywords: Light-chain amyloidosis; staging system; hepatic function; renal function

Submitted Jul 22, 2021. Accepted for publication Aug 16, 2021.

doi: 10.21037/atm-21-4033

View this article at: https://dx.doi.org/10.21037/atm-21-4033

\section{Introduction}

Systemic light-chain (AL) amyloidosis is triggered by underlying clonal plasma cell dyscrasia that produces amyloidogenic immunoglobulin light chains, resulting in the deposition of diffuse misfolded protein fibrils, which can lead to fatal functional damage to multiple vital organs (1). AL amyloidosis is a rare disease with a 3 -year overall survival (OS) rate of only $41 \%$ if left untreated (2).

^ ORCID: Wei Yan, 0000-0002-6305-1011; Aijun Liao, 0000-0001-7631-6157; Wei Yang, 0000-0003-2945-2898; Huihan Wang, 0000$0003-1141-5263$. 
Disease prognosis is strongly associated with the severity of organ involvement, especially cardiac, renal, and hepatic involvement $(3,4)$, which is usually a cause of substantial morbidity and often results in rapidly progressive organ failure and death.

The current staging systems use markers of myocardial cell injury and heart failure, as these are highly sensitive to cardiac involvement in amyloidosis (5). The Mayo Clinic 2004 staging system (Mayo 2004) uses cardiac troponin $\mathrm{T}$ (cTnT) and $\mathrm{N}$-terminal pro-brain natriuretic peptide (NT-proBNP) to classify patients into three stages (6). The Mayo 2012 system groups patients according to cTnT or high-sensitivity troponin T, NT-proBNP, and the value of the difference between the involved and uninvolved free light chain (dFLC) (7). Cardiac involvement greatly affects the disease outcome; therefore, markers of cardiac injury and dysfunction are powerful prognostic factors in $\mathrm{AL}$ amyloidosis (8). However, AL amyloidosis is a systemic disease characterized by the deposition of unique fibrils in different vital organs (9). Thus, in addition to cardiac involvement, hepatic and renal involvement can also affect long-term survival or lead to early death. Palladini et al. designed a staging system that uses proteinuria and the glomerular filtration rate to discriminate patients at risk of progression to dialysis (10). Proteinuria $>5 \mathrm{~g} / 24 \mathrm{~h}$ and an estimated glomerular filtration rate (eGFR) $<50 \mathrm{~mL} / \mathrm{min}$ were adopted for predicting the progression to dialysis (10). According to a retrospective analysis of the clinical features and natural history data of 102 patients diagnosed with systemic AL amyloidosis with hepatic involvement, increased total bilirubin (Tbil) indicates reduced progressionfree survival and OS (4). The majority of patients have a significant impairment of vital organs, the existing staging systems do not consider the simultaneous inclusion of multiple organ function indicators. Therefore, factors that can better reflect the effects of multiple organ involvement (including renal and hepatic involvement) on the survival of patients with AL amyloidosis should be explored for potential inclusion in a prognostic disease staging system.

Here, we demonstrate a novel staging system based on a nationwide survey of 1,064 patients in China. This novel staging system incorporates serum indicators reflecting organ function as well as the level of amyloidogenic lightchain synthesis. Therefore, it could be used to explain the heterogeneity in the outcomes of patients with $\mathrm{AL}$ amyloidosis with multiple organ involvement. We present the following article in accordance with the TRIPOD reporting checklist (available at https://dx.doi.org/10.21037/ atm-21-4033).

\section{Methods}

\section{Patients}

With the cooperation of 18 hospitals in the Chinese Registration Network for Light-chain Amyloidosis, a nationwide survey was conducted from 2009 to 2020, and 1,064 patients with systemic AL amyloidosis were registered and followed. The diagnosis of systemic AL amyloidosis was confirmed when Congo red-positive fibril deposition was observed, and the deposits were characteristic of the AL type according to immunohistochemistry, immunofluorescence, or laser microdissection with mass spectrometry-based proteomic analysis. To construct the staging system, a retrospective analysis was performed with the clinical and laboratory data of 813 patients who were also classifiable according to the current Mayo 2012 staging system. A validation cohort of 251 patients was used for further evaluation of the new prognostic staging system. Treatment options included autologous stem cell transplantation (ASCT), bortezomib-based therapy, a Thalidomide-based regimen, a melphalan-based regimen, and others. The study was performed according to the principles of the Declaration of Helsinki (as revised in 2013) and approved by the Medical Ethics Committee of the Shengjing Hospital of China Medical University (2020PS055J). All participants provided written informed consent.

\section{Follow-up}

We followed up all patients at the clinics or via telephone before recording the follow-up data. The final follow-up date was October 31,2020. OS was defined as the time from diagnosis to death or the last follow-up. Patients who were alive at the last follow-up were censored at that date. Progression-free survival was defined as the period from treatment initiation to disease progression, relapse, or death from any cause.

\section{Statistical analysis}

A receiver operating characteristic curve analysis, with calculation of the Youden Index, was performed to determine the cut-off values. The prognostic factors for OS were identified based on a Cox proportional hazards 
analysis. Survival curves were constructed using the Kaplan-Meier method and compared using a log-rank test. Differences in nominal variables between groups were tested using Fisher's exact test, and differences in continuous variables between groups were determined using the Wilcoxon signed-rank test. Missing data were handled with multiple imputations. RealMedSci software (Shenyang, China) was used for the statistical analyses.

\section{Results}

The baseline characteristics of the 1,064 patients in this study are presented in Table 1 . The patients had a median age of 59 years (range, 46-70 years), and 665 (63\%) were male. The median follow-up duration in the whole test cohort was 14.5 months (range, 0.1-146 months). According to the Kaplan-Meier analysis, the median survival time from baseline was 25.3 months [95\% confidence interval (CI), 24-27.9 months]. A total of 354 patients died during the follow-up period.

\section{Identification of prognostic variables}

Organ function-related characteristics were considered, including proteinuria, albumin, eGFR, Tbil, cardiac troponin I (cTnI), and NT-proBNP. Of these, cTnI, NT-proBNP, and eGFR have been identified previously as being prognostically valuable in AL amyloidosis. Additionally, dFLC was included as a plasma cell clonerelated characteristic. The hazard ratios related to these variables in the univariate and multivariate analyses are shown in Table 2. All variables, except for proteinuria, were significant predictors of OS in the univariate analysis. In the multivariate analysis model, eGFR, Tbil, cTnI, NTproBNP, and dFLC were independent prognostic factors. The influence weights of all parameters on prognosis were similar, with NT-proBNP having the largest and cTnI the smallest.

\section{Development of the prognostic model}

A score of 1 was assigned for each of the following five prognostic variables: eGFR $<60 \mathrm{~mL} / \mathrm{min} / 1.73 \mathrm{~m}^{2}$, Tbil $\geq 18 \mu \mathrm{mol} / \mathrm{L}$, cTnI $\geq 0.06 \mu \mathrm{g} / \mathrm{L}, \mathrm{NT}$-proBNP $\geq 3,600 \mathrm{pg} / \mathrm{mL}$, and $\mathrm{dFLC} \geq 100 \mathrm{mg} / \mathrm{L}$. The patients were grouped into five disease stages (0, I, II, III, and IV) according to scores of $0,1,2,3$, and $4-5$, respectively. The number of patients with stage 0, I, II, III, and IV disease was 220 (20.7\%),
291 (27.3\%), 251 (23.6\%), 178 (16.7\%), and 124 (11.7\%), respectively.

The median OS for those with stage II, III, and IV disease was 56.9 months [95\% CI, 33.9-not reached (NR)], 18.6 months (95\% CI, 33.9-NR), and 6.5 months (95\% CI, 8.0-24.6), respectively $(\mathrm{P}<0.001)$. The 3 -year survival estimates for those with stage 0 , I, II, III, and IV disease were $90.7 \%, 71.4 \%, 59.4 \%, 39.0 \%$, and $22.1 \%$, respectively (Figure 1).

\section{Comparison with the Mayo 2012 staging system}

We examined how the new system would group patients in each of the five stages as defined by the Mayo 2012 system based on cTnI, NT-proBNP, and dFLC. The patients, grouped according to the Mayo 2012 system, were further subdivided according to the new system, and their median OS outcomes are listed in Table 3. This analysis showed that the new system was able to identify patients with different outcomes compared to the classic-stage groups.

\section{Application of the new staging system to subgroups of patients with cardiac, hepatic, and renal involvement}

Subgroups of patients with cardiac, hepatic, and renal involvement were examined. The number of patients with renal involvement only, cardiac involvement only, and hepatic involvement only was 205 (19.2\%), 157 $(14.8 \%)$, and $9(0.84 \%)$, respectively. The number of patients with multiple organ involvement, including renal involvement, cardiac involvement, and hepatic involvement was $793(74 \%), 710(67 \%)$, and 190 (18\%), respectively. Subsequently, we examined how the new system would classify these patients in every stage of the Mayo 2012 staging system. Table 4 shows the number and median OS outcomes of patients with renal involvement only, cardiac involvement only, and hepatic involvement who were divided based on the Mayo 2012 system and then subdivided based on the new system; Figure 2 shows the survival probability of the same groups plotted against time.

\section{Validation of the prognostic model}

Next, the staging system was verified in the validation cohort of 251 patients. Of these, 51 (20.3\%), 65 (25.9\%), 66 (26.3\%), 40 (15.9\%), and 29 (11.6\%) had stage 0, I, II, III, and IV disease, respectively. The patients with stage II, III, and IV disease had a median OS of 36.5 months (95\% CI, 
Table 1 Baseline demographics and characteristics of the 1,064 patients

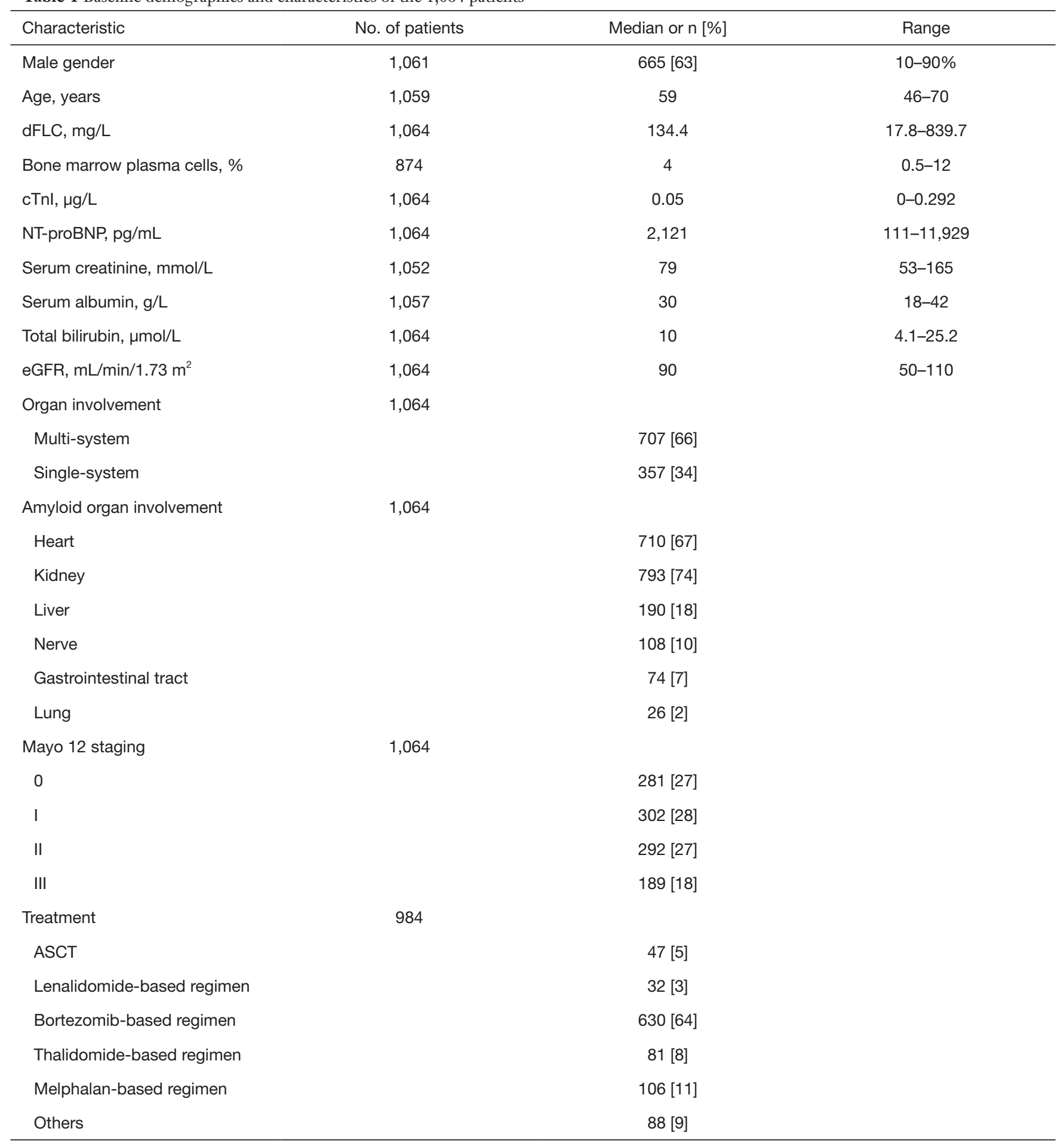

dFLC, difference between involved and uninvolved light chain; cTnl, cardiac troponin I; NT-proBNP, N-terminal fragment of the prohormone brain natriuretic peptide; eGFR, estimated glomerular filtration rate; ASCT, autologous stem cell transplantation. 
Table 2 Results of univariate and multivariate analyses of various prognostic factors

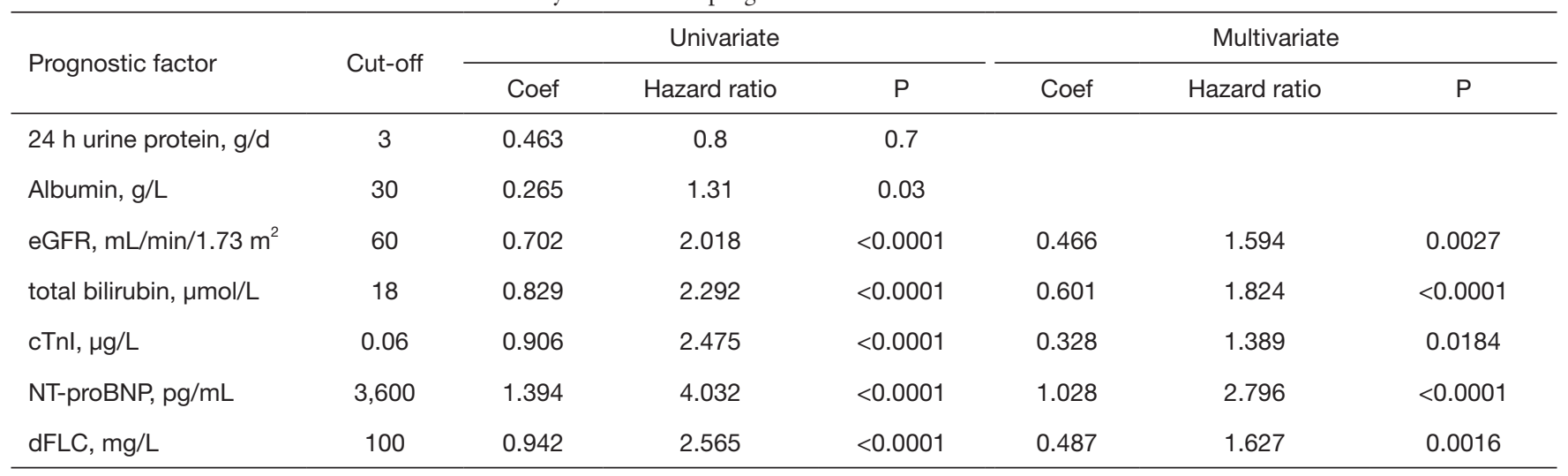

dFLC, difference between involved and uninvolved light chain; cTnl, cardiac troponin I; NT-proBNP, N-terminal fragment of the prohormone brain natriuretic peptide; eGFR, estimated glomerular filtration rate; coef, coefficient.

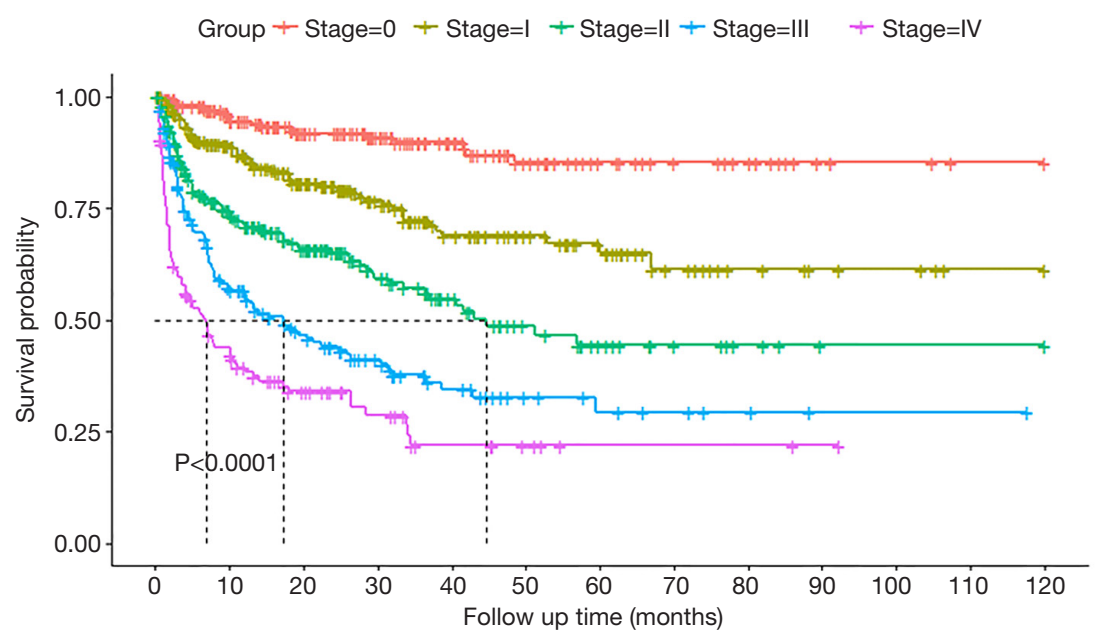

Figure 1 Kaplan-Meier curves for OS among the test cohort $(\mathrm{n}=813)$ patients based on the new staging system. OS, overall survival.

26.4-NR), 9.7 months (95\% CI, 3.5-30.4), and 6.8 months (95\% CI, 3.5-30.4), respectively $(\mathrm{P}<0.001)$. The 3 -year survival estimates for patients with stage $0, \mathrm{I}, \mathrm{II}, \mathrm{III}$, and IV disease were $87.6 \%, 75.0 \%, 52.0 \%, 33.1 \%$, and $20.4 \%$, respectively (Figure $\mathrm{S} 1)$.

\section{Discussion}

The current study developed and validated a new staging system for AL amyloidosis based on data from the largest cohort to date. Of note, unlike prior staging systems, the new system incorporates plasma cell-related characteristics in addition to prognostic laboratory markers related to cardiac, renal, and hepatic function.

$\mathrm{AL}$ amyloidosis is a plasma cell dyscrasia (11) in which pathogenic plasma cells secrete an excess amount of amyloidogenic light chains that are conformationally changed and subsequently cause amyloid fibrils $(12,13)$. Organ architecture disruption, together with the direct cytotoxic effect of light chains, results in organ dysfunction (14). Kumar et al. evaluated plasma cell clone-related characteristics, namely dFLC, maximum bone marrow plasma cell percentage, $\beta 2$-microglobulin, plasma cell labeling index, and the presence or absence of circulating plasma cells, and revealed that of these, serum FLC is prognostically valuable in AL amyloidosis (15). A high $\mathrm{dFLC}$ is related to more severe organ dysfunction and an unfavorable prognosis $(16,17)$. A threshold dFLC of $\geq 180 \mathrm{mg} / \mathrm{L}$ is well-validated and used as part of the Mayo 2012 AL amyloidosis staging system. In our new 
Table 3 Comparison of the new and Mayo 2012 staging systems

\begin{tabular}{|c|c|c|c|c|c|c|c|c|}
\hline New stage & \multicolumn{2}{|c|}{ Mayo stage I } & \multicolumn{2}{|c|}{ Mayo stage II } & \multicolumn{2}{|c|}{ Mayo stage III } & \multicolumn{2}{|c|}{ Mayo stage IV } \\
\hline Total & 281 & NR & 302 & NR & 292 & $37.0(26.1-\mathrm{NR})$ & 189 & $10.8(7.3-26.3)$ \\
\hline 0 & 192 & NR & 28 & NR & 0 & - & 0 & - \\
\hline III & 0 & - & 14 & 17.3 (6.5-NR) & 88 & $17.6(8.0-31.4)$ & 76 & 15.2 (7.8-NR) \\
\hline IV & 0 & - & 0 & - & 39 & 7.1 (4.1-NR) & 85 & $5.0(2.3-10.6)$ \\
\hline
\end{tabular}

OS, overall survival; $\mathrm{Cl}$, confidence interval; NR, not reached.

Table 4 Comparison of the new and Mayo 2012 staging systems of patients with multiple organ involvement

\begin{tabular}{|c|c|c|c|c|c|c|c|c|}
\hline & \multicolumn{2}{|r|}{ Mayo I stage } & \multicolumn{2}{|r|}{ Mayo II stage } & \multicolumn{2}{|r|}{ Mayo III stage } & \multicolumn{2}{|r|}{ Mayo IV stage } \\
\hline \multicolumn{9}{|c|}{ Heart involvement only $(n=157)$} \\
\hline Total & 5 & 33.5 (33.5-NR) & 43 & NR & 65 & $21.3(8.8-N R)$ & 44 & $10.2(6.9-N R)$ \\
\hline Stage 0 & 3 & NR & 5 & NR & 0 & - & 0 & - \\
\hline Stage II & 0 & - & 14 & NR & 18 & $43.0(7.1-\mathrm{NR})$ & 7 & NR \\
\hline Stage III & 0 & - & 4 & $4.9(1.2-N R)$ & 26 & 20.4 (9.7-NR) & 17 & 59.4 (7.3-NR) \\
\hline Stage IV & 0 & - & 0 & - & 14 & $7.0(2.0-\mathrm{NR})$ & 20 & $6.5(2.0-N R)$ \\
\hline \multicolumn{9}{|c|}{ Kidney involvement only ( $\mathrm{n}=205)$} \\
\hline Stage I & 38 & NR & 46 & NR & - & - & - & - \\
\hline Stage II & 1 & NR & 19 & $41.6(40.6-N R)$ & - & - & - & - \\
\hline Stage III & 0 & - & - & - & - & - & - & - \\
\hline Stage IV & 0 & - & - & - & - & - & - & - \\
\hline \multicolumn{9}{|c|}{ Liver involvement $(n=190)$} \\
\hline Total & 46 & NR & 53 & NR & 54 & $12.1(4.9-38.7)$ & 37 & $3.8(1.6-N R)$ \\
\hline Stage 0 & 25 & NR & 4 & NR & 0 & - & 0 & - \\
\hline Stage I & 16 & NR & 14 & NR & 6 & 37 (37.0-NR) & 0 & - \\
\hline
\end{tabular}

OS, overall survival; Cl, confidence interval; NR, not reached. 
A

Group + Stage $=0+$ Stage $=I+$ Stage $=I I+$ Stage $=I I I+$ Stage $=I V$

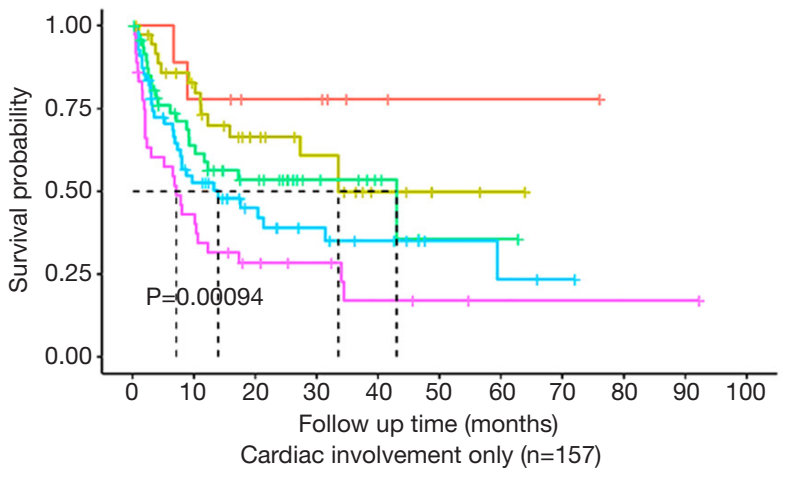

C

Group + Stage $=0+$ Stage $=1+$ Stage $=I I+$ Stage $=I I I+$ Stage $=$ IV

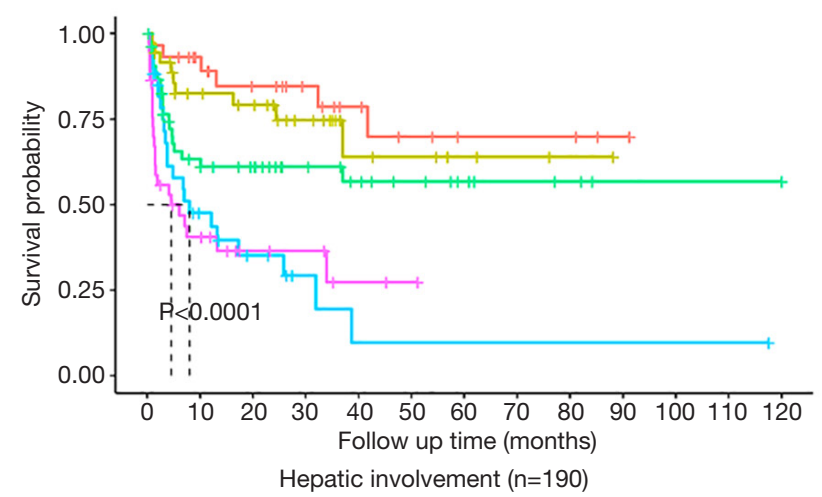

B

Group + Stage $=0+$ Stage $=1+$ Stage $=\|$

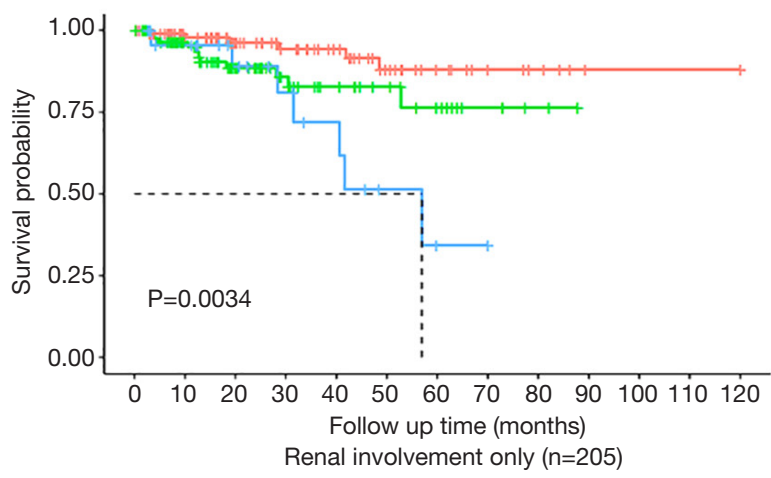

Figure 2 Kaplan-Meier curves for OS among patients with different organ involvement based on the new staging system. (A) cardiac involvement only ( $\mathrm{n}=157)$, (B) renal involvement only $(\mathrm{n}=205)$, and $(\mathrm{C})$ hepatic involvement $(\mathrm{n}=190)$. OS, overall survival.

staging system, based on the data from 1,064 patients with AL amyloidosis, dFLC was also a prognostic factor at a cut-off value of $\geq 100 \mathrm{mg} / \mathrm{L}$.

As a multisystemic disorder, AL amyloidosis can affect the heart, kidneys, liver, nerves, lungs, and bowel. Common management approaches include anti-plasma cell systemic chemotherapy and high-dose chemotherapy followed by ASCT; however, only $20-25 \%$ of patients with AL amyloidosis are considered eligible for stem cell transplantation based on age, renal function, and severity of heart failure (18-20). Effective therapy exists, although it is ineffective if the end-organ damage is severe. In almost $40 \%$ of patients, advanced organ involvement is observed in the first year of diagnosis, and $25 \%$ of patients die within 6 months of diagnosis (21). Commonly, the early outcome is closely related to the range and severity of organ involvement (22). Therefore, it is vital for clinicians to accurately estimate the prognosis of $\mathrm{AL}$ amyloidosis based on organ involvement.

The morbidity and mortality of patients with AL amyloidosis are closely related to the severity of cardiac involvement. The Mayo 2004 system classifies patients into three stages according to the following threshold values: cTnT $\geq 0.035 \mathrm{~g} / \mathrm{L}, \mathrm{c} T n \mathrm{I} \geq 0.1 \mathrm{~g} / \mathrm{L}$, and NT-proBNP $\geq 332 \mathrm{ng} / \mathrm{L}$. Moreover, the Mayo 2012 model classifies patients based on cTnT $\geq 0.025 \mathrm{ng} / \mathrm{mL}$ and NT-proBNP $\geq 1,800 \mathrm{pg} / \mathrm{mL}$. Cardiac troponins provide an indication of myocardial damage. In patients with normal renal function, cTnT levels will increase in the first 3 to 5 hours following myocardial infarction and remain high for up to 21 days, whereas their cTnI levels will increase in the first 3 hours and remain high for up to 7 days. According to recent studies, serum cardiac troponin levels are elevated in at least $50 \%$ of patients with renal disease (23). Therefore, for patients with amyloidosis who only have renal involvement, it remains unclear whether troponin reflects the degree of 
renal involvement and the prognosis. Thus, we included the eGFR as a factor reflecting renal function in our staging system to supplement the assessment of amyloidosis in patients with multiple organ involvement.

The kidney is the second most frequently involved organ in patients with AL amyloidosis (24). Previously, a staging system based on the combination of proteinuria and eGFR was proposed to estimate the risk of end-stage renal disease; the prognostic value of eGFR $\left(<50 \mathrm{~mL} / \mathrm{min} / 1.73 \mathrm{~m}^{2}\right)$ and proteinuria $(>5 \mathrm{~g} / \mathrm{d})$ at diagnosis for predicting dialysis was confirmed (25), and these results might be used as guidelines in clinical treatment. In our new staging system, we included eGFR $<60 \mathrm{~mL} / \mathrm{min} / 1.73 \mathrm{~m}^{2}$ to improve the evaluation of patients with $\mathrm{AL}$ amyloidosis with only renal involvement. Of course, eGFR is also affected by cardiac function; hence, sometimes eGFR alone cannot be used to evaluate patients' renal function. That said, our staging system also includes indicators of cardiac function, and the combination of the two can better stratify patient risk.

Patients with hepatic systemic AL amyloidosis may have mild symptoms such as abdominal distension, weight loss, and early satiety, occasionally followed by hepatosplenomegaly, jaundice, and ascites. Severe liver involvement in $\mathrm{AL}$ amyloidosis is assumed to affect $\mathrm{OS}$ adversely. However, available biomarkers for quantifying hepatic involvement (e.g., serum gamma-glutamyl transferase, alkaline phosphatase, and FibroScan) are limited compared to markers for cardiac and renal involvement in the existing staging systems. In our new staging system, we found that $\mathrm{Tbil} \geq 18 \mu \mathrm{mol} / \mathrm{L}$ might be a valuable indicator of hepatic involvement in patients with AL amyloidosis. Although elevated levels of alkaline phosphatase and gamma-glutamyl transferase were frequently observed in patients with AL amyloidosis with hepatic involvement, they did not have a sufficient effect on patient outcomes in our study.

The current staging system was developed using the largest cohort $(n=1,064)$ of patients with $\mathrm{AL}$ amyloidosis to date, with a long-term follow-up. The Mayo 2012 staging system was applied to classify 813 patients in the test cohort, and the median OS of the patients in Mayo 2012 stages I and II was NR, suggesting a good prognosis for these patients. However, these two groups were further classified using our new staging system, and the results showed that the median OS of patients with new stage II from Mayo 2012 stage I was 37 months while that of patients with new stage III from Mayo 2012 stage II was 17.3 months; thus, the prognosis was not satisfactory. Using our novel system, patients with worse outcomes can be identified from those grouped in stages I and II according to the previous staging system. One reason for the discrepancy in staging between the systems could be that these patients had severe hepatic and renal function damage without obvious cardiac amyloidosis. Notably, patients with only hepatic and renal involvement may be classified into stage I or II using the Mayo 2012 staging system while having a poor prognosis. Our new staging system, which includes two additional indicators compared to the classic Mayo staging system, is more complex to use; however, it may be more advantageous for patients with multiple organ involvement.

In conclusion, a new staging system has been developed that incorporates plasma cell-related characteristics in addition to laboratory markers of prognosis based on cardiac, renal, and hepatic function. This staging system for AL amyloidosis will allow clinicians to better classify patients with multiple organ involvement.

\section{Acknowledgments}

Funding: None.

\section{Footnote}

Reporting Checklist: The authors have completed the TRIPOD reporting checklist. Available at https://dx.doi. org/10.21037/atm-21-4033

Data Sharing Statement: Available at https://dx.doi. org/10.21037/atm-21-4033

Conflicts of Interest: All authors have completed the ICMJE uniform disclosure form (available at https://dx.doi. org/10.21037/atm-21-4033). The author report that this work was supported by the Neusoft Research of Intelligent Healthcare Technology, Co., Ltd. The authors are grateful for their support in terms of data extraction, analysis, and evaluation by leveraging RealOne ISCI and RealMedSci and for allowing us to use their clinic research platform and automatic medical analysis platform. This work was also supported by the Chinese Registration Network for Lightchain Amyloidosis. The authors have no other conflicts of interest to declare.

Ethical Statement: The authors are accountable for all aspects of the work in ensuring that questions related to the accuracy or integrity of any part of the work are 
appropriately investigated and resolved. The study was performed according to the principles of the Declaration of Helsinki (as revised in 2013) and approved by the Medical Ethics Committee of the Shengjing Hospital of China Medical University (2020PS055J). All participants provided written informed consent.

Open Access Statement: This is an Open Access article distributed in accordance with the Creative Commons Attribution-NonCommercial-NoDerivs 4.0 International License (CC BY-NC-ND 4.0), which permits the noncommercial replication and distribution of the article with the strict proviso that no changes or edits are made and the original work is properly cited (including links to both the formal publication through the relevant DOI and the license). See: https://creativecommons.org/licenses/by-nc-nd/4.0/.

\section{References}

1. Gertz MA. Immunoglobulin light chain amyloidosis: 2018 Update on diagnosis, prognosis, and treatment. Am J Hematol 2018;93:1169-80.

2. Zhao Q, Li F, Song P, et al. Clinical Characteristics and Treatment Outcome of Chinese Patients With Systemic Amyloid Light-Chain Amyloidosis: A Retrospective Single-Center Analysis. Clin Lymphoma Myeloma Leuk 2016;16:104-10.

3. Gertz MA. Immunoglobulin light chain amyloidosis: 2020 update on diagnosis, prognosis, and treatment. Am J Hematol 2020;95:848-60.

4. Zhang LL, Shen KN, Zhang CL, et al. Clinical presentation and prognostic analysis of Chinese patients with systemic light chain amyloidosis with liver involvement. Leuk Res 2019;86:106226.

5. Martinez-Naharro A, Hawkins PN, Fontana M. Cardiac amyloidosis. Clin Med (Lond) 2018;18:s30-5.

6. Dispenzieri A, Gertz MA, Kyle RA, et al. Serum cardiac troponins and $\mathrm{N}$-terminal pro-brain natriuretic peptide: a staging system for primary systemic amyloidosis. J Clin Oncol 2004;22:3751-7.

7. Kumar S, Dispenzieri A, Lacy MQ, et al. Revised prognostic staging system for light chain amyloidosis incorporating cardiac biomarkers and serum free light chain measurements. J Clin Oncol 2012;30:989-95.

8. Rubin J, Maurer MS. Cardic amyloidosis: overlooked, underappreciated, and treatable. Annu Rev Med 2020;71:203-19.

9. Dittrich T, Kimmich C, Hegenbart U, et al. Prognosis and staging of AL amyloidosis. Acta Haematol 2020;143:388-400.

10. Palladini G, Hegenbart U, Milani P, et al. A staging system for renal outcome and early markers of renal response to chemotherapy in $\mathrm{AL}$ amyloidosis. Blood 2014;124:2325-32.

11. Huang XF, Jian S, Lu JL, et al. Genomic profiling in amyloid light-chain amyloidosis reveals mutation profiles associated with overall survival. Amyloid 2020;27:36-44.

12. Bhutani D, Leng S, Lentzsch S. Fibril-directed Therapies in Systemic Light Chain AL Amyloidosis. Clin Lymphoma Myeloma Leuk 2019;19:555-9.

13. Blancas-Mejia LM, Misra P, Dick CJ, et al. Assays for Light Chain Amyloidosis Formation and Cytotoxicity. Methods Mol Biol 2019;1873:123-53.

14. Blancas-Mejia LM, Misra P, Dick CJ, et al. Immunoglobulin light chain amyloid aggregation. Chem Commun (Camb) 2018;54:10664-74.

15. Kumar S, Dispenzieri A, Katzmann JA, et al. Serum immunoglobulin free light-chain measurement in primary amyloidosis: prognostic value and correlations with clinical features. Blood 2010;116:5126-9.

16. Sanchorawala V, Seldin DC, Magnani B, et al. Serum free light-chain responses after high-dose intravenous melphalan and autologous stem cell transplantation for AL (primary) amyloidosis. Bone Marrow Transplant 2005;36:597-600.

17. Mollee P, Merlini G. Free light chain testing for the diagnosis, monitoring and prognostication of AL amyloidosis. Clin Chem Lab Med 2016;54:921-7.

18. Varga C, Titus SE, Toskic D, et al. Use of novel therapies in the treatment of light chain amyloidosis. Blood Rev 2019;37:100581.

19. Li G, Han D, Wei S, et al. Multiorgan involvement by amyloid light chain amyloidosis. J Int Med Res 2019;47:1778-86.

20. Palladini G, Comenzo RL. The challenge of systemic immunoglobulin light-chain amyloidosis (Al). Subcell Biochem 2012;65:609-42.

21. Cohen OC, Sharpley F, Gillmore JD, et al. Use of ixazomib, lenalidomide and dexamethasone in patients with relapsed amyloid light-chain amyloidosis. $\mathrm{Br} \mathrm{J}$ Haematol 2020;189:643-9.

22. Kourelis TV, Kumar SK, Go RS, et al. Immunoglobulin light chain amyloidosis is diagnosed late in patients with preexisting plasma cell dyscrasias. Am J Hematol 2014;89:1051-4. 
Page 10 of 10

23. Donaldson A, Cove-Smith R. Cardiac troponin levels in patients with impaired renal function. Hosp Med 2001;62:86-9.

24. Kufová Z, Sevcikova T, Growkova K, et al. Biomarkers in Immunoglobulin Light Chain Amyloidosis. Klin Onkol Summer;30:60-7.

Cite this article as: Yan W, Cao Y, Liao A, Yang W, Li J, Wang H. A prognostic staging system for light-chain amyloidosis using hepatic and renal indicator data from 1,064 Chinese patients. Ann Transl Med 2021;9(16):1347. doi: 10.21037/atm-21-4033
Yan et al. New prognostic staging system for $A L$ amyloidosis

25. Zhu Z, Yue C, Sun Y, et al. Light-chain amyloidosis with renal involvement: renal outcomes and validation of two renal staging systems in the Chinese population. Amyloid 2019;26:186-91.

(English Language Editor: D. Fitzgerald) 


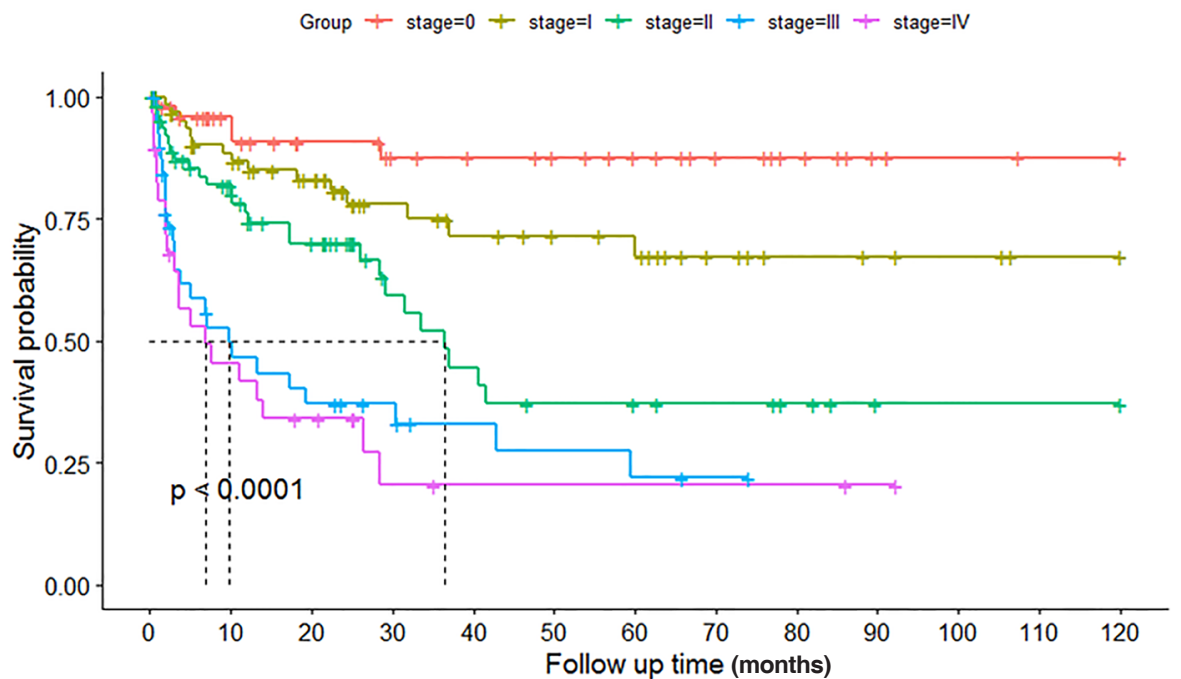

Figure S1 Kaplan-Meier curves for OS among the validation cohort $(\mathrm{n}=251)$ patients based on the new staging system. 